\title{
A high-protein diet increases faecal IgA concentrations in baby pigs
}

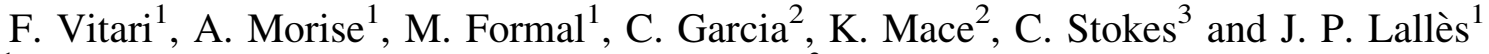 \\ ${ }^{1}$ INRA, UMR1079 SENAH, 35590, Saint-Gilles, France, ${ }^{2}$ Nestlé Research Center, Lausanne, Switzerland \\ and ${ }^{3}$ Veterinary School, Langford, University of Bristol, Bristol, UK
}

Feeding low-birth-weight (BW) babies with high-protein formulas is common practice for catching up a normal body weight. However, epidemiological data have suggested that this practise increases the risk of obesity in adolescence ${ }^{(1)}$. The influence of such diets on intestinal immunity is not known. IgA secreted into the gastrointestinal tract is essential for its protection against infections ${ }^{(2)}$. The aim of the present study was to compare the effects of normal (NP)- and high (HP)-protein formulas and maternal milk (MM) on ileal and faecal IgA in the swine model, using low- and normal-BW pigs.

Eighty suckling pigs (forty low BW and forty normal BW; 0.9 v. 1.4 (SE 0.03) kg respectively) were assigned to four treatments (MM-7d, MM-28d, NP and HP) at $7 \mathrm{~d}$ of age according to incomplete intra-litter blocks. MM pigs were kept with the sow and killed at 7 or $28 \mathrm{~d}$ of age (MM-7d and MM-28d respectively). NP and HP pigs were placed in metabolism cages individually and fed formulas until death at day 28. The NP formula was designed to mimic sow's milk composition while the HP formula contained $33 \%$ (w/w) more protein. Ileal digesta and faeces and blood plasma were collected after death and frozen at $-20^{\circ} \mathrm{C}$ until IgA analysis by ELISA. Data were analysed statistically using the GLM procedure of SAS (SAS Institute Inc., Cary NC, USA) after log transformation. The model included birth weight, block, litter, age at slaughter, diet and relevant interactions. Groups were compared by pre-planned non-orthogonal contrasts when the diet effect was significant $(P<0.05)$.

The effects of litter, BW and interactions were not significant $(P>0.05)$. Ileal IgA decreased between 7 and $28 \mathrm{~d}$ of age $(P<0.05)$ in MM piglets. The feeding regimen had no influence on ileal $\operatorname{IgA}$ at $28 \mathrm{~d}$. IgA concentration in faeces did not vary between 7 and $28 \mathrm{~d}$ in MM piglets. At $28 \mathrm{~d}$ of age faecal IgA was higher in HP-fed pigs than in NP-fed pigs $(P<0.05)$, with IgA levels being intermediate in MM pigs. Plasma IgA at $28 \mathrm{~d}$ (not determined at $7 \mathrm{~d}$ ) was higher in formula-fed piglets as compared with MM piglets $(P<0.05)$ with no difference between NP and HP diets.

The HP diet increased faecal IgA concentration in young pigs regardless of BW. This effect may reflect an increased IgA response to dietary antigens. The lack of diet effect at the ileal level suggests a role of the large intestine, despite the fact that the small intestine is the major site of IgA production ${ }^{(3)}$. Underlying mechanisms may involve a stimulation of colonic $\operatorname{IgA}$ production, a reduction in $\operatorname{IgA}$ degradation in the colon or both. In contrast, such an effect of the diet was not seen systemically, indicating a specific intestinal response. Decreased ileal IgA with increasing age may be a result of a high passive immunity after colostrum consumption during the first days of life. Finally, feeding formulas appeared to stimulate systemic immunity.

1. Rolland-Cachera MF, Deheeger M, Akrout M \& Bellisle F (1995) Int J Obes Relat Metab Disord 19, 573-578.

2. Welsh JK \& May JT (1979) J Pediatr 94, 1-9.

3. Danis VA, Harries AD \& Heatley RV (1984) Clin Exp Immunol 5, 159-166. 